\title{
BMVC99
}

\section{Curvature Estimation on Smoothed 3-D Meshes}

\author{
Peter Yuen, Nasser Khalili and Farzin Mokhtarian
}

\author{
Centre for Vision, Speech and Signal Processing \\ School of Electronic Engineering, Information Technology and Mathematics \\ University of Surrey, Guildford, GU2 5XH, UK \\ Tel: +44 1483 876035, Fax: +44-1483-259554 \\ Email: $\{$ P. Yuen, N.Khalili, F.Mokhtarian\}@ee.surrey.ac.uk \\ http://www.ee.surrey.ac.uk/Research/VSSP/demos/css3d/index.html
}

\begin{abstract}
A novel technique for multi-scale curvature computation on a smoothed 3D surface is presented. In our technique, semigeodesic coordinates are constructed at each vertex of the mesh which becomes the local origin. A geodesic from the origin is first constructed in an arbitrary direction such as the direction of one of the incident edges. The surface Gaussian and mean curvatures are then estimated. Next, the curvature zero-crossing contours were recovered. Curvature features such as zero-crossing contours and maxima recovered at multiple scales are useful for surface matching and object recognition algorithms, as well as registration of 3-D medical data. The performance of our technique when selecting different directions as an arbitrary direction for the geodesic at each vertex is also evaluated. Our experiments demonstrate that estimation of smoothed surface curvatures are very accurate and not affected by the arbitrary direction of the first geodesic line when constructing semigeodesic coordinates. Our technique is independent of the underlying triangulation and is also more efficient than volumetric diffusion techniques since 2-D rather than 3-D convolutions are employed.
\end{abstract}

\section{Introduction}

This paper introduces a new technique for multi-scale curvature computation on a smoothed 3-D surface. Complete triangulated models of 3-D objects are constructed and using a local parametrisation technique, are then smoothed using a 2-D Gaussian filter. The technique considered here is a generalization of earlier multi-scale representation theories proposed for 2-D contours [9] and space curves [7]. More details of the diffusion technique as well as literature survey appear in [8].

Some evolution properties of 3-D surfaces are described in this paper. The surface Gaussian and mean curvatures were estimated and the curvature zero-crossing contours were recovered and displayed on the surface. Curvature features such as zero-crossing contours and maxima recovered at multiple scales are useful for surface matching and object recognition algorithms, as well as registration of 3-D medical data. The performance of our technique when selecting different directions as an arbitrary direction for 


\section{BMVC99}

the construction of semigeodesic coordinates, are also presented and results indicate that estimation of smoothed surface curvatures are very accurate and not affected by the arbitrary direction of the first geodesic line when constructing semigeodesic coordinates. The error observed for the estimation of Gaussian curvature is smaller than that of the mean curvature and diffusion reduces the estimation error of both surface curvatures.

\section{Literature Survey}

This section presents a survey of previous work in representation of 3-D surfaces. Comprehensive surveys of 3-D object recognition systems are presented by Besl and Jain [1]. Sinha and Jain [13] provide an overview of geometry based representations derived from range data of objects. Generalised cones or cylinders [14] as well as geons [10] approximate a 3-D object using globally parametrised mathematical models, but they are not applicable to detailed free-form objects. A form of 3-D surface smoothing has been carried out in $[15,16]$ but this method has drawbacks since it is based on weighted averaging using neighbouring vertices and is therefore dependent on the underlying triangulation. In volumetric diffusion $[6,5]$ or level set methods [12], an object is treated as a filled area or volume. The major shortcoming of these approaches is that the entire object data must be available. This problem makes them unsuitable for object recognition in presence of occlusion.

\section{Semigeodesic Parametrisation}

Free-form 3-D surfaces are complex hence, no global coordinate system exists on these surfaces which could yield a natural parametrisation of that surface. Studies of local properties of 3-D surfaces are carried out in differential geometry using local coordinate systems called curvilinear coordinates or Gaussian coordinates [2]. Each system of curvilinear coordinates is introduced on a patch of a regular surface referred to as a simple sheet. A simple sheet of a surface is obtained from a rectangle by stretching, squeezing, and bending but without tearing or gluing together. Given a parametric representation $\mathbf{r}=\mathbf{r}(u, v)$ on a local patch, the values of the parameters $u$ and $v$ determine the position of each point on that patch. Construction and implementation of semigeodesic coordinates in our technique is described in $[8,4]$.

\subsection{Arbitrary Geodesic Line}

Before the semigeodesic coordinates can be generated on a local patch at a chosen vertex $V$, an arbitrary geodesic line is required. Since the direction of the arbitrary geodesic line can be randomly selected, the edge connecting $V$ and one of its neighbours is selected as the arbitrary direction. Once the arbitrary direction is determined, the next step is to construct a geodesic line and hence semigeodesic coordinates at each vertex of the 3-D triangular mesh. During the diffusion process, a 3-D surface is also sampled locally at a specified step size. This process can be slowed down by reducing the step size or it can be speeded up by increasing the step size. 


\section{BMVC99}

\section{Evolution Properties of 3-D Surfaces}

Before we present the results on curvature estimation on 3-D surfaces, the following theorems and their proofs on fundamental properties of evolution will be presented in this section.

Theorem I: The order of application of evolution and a shape preserving transformation to a surface does not change the final result.

Proof: Suppose surface $\mathcal{S}$ is evolved into $S_{\sigma}$. Every point of $S_{\sigma}$ is a weighted average of a subset of points of $\mathcal{S}$. Therefore evolution at each point $Q$ of $\mathcal{S}$ can be expressed as the convolution of a neighbourhood of $Q$ with a 2-D function (not Gaussian) with unknown values.

$$
P(X, Y, Z)=(x(u, v) \otimes f(u, v), y(u, v) \otimes f(u, v), z(u, v) \otimes f(u, v))
$$

$\otimes$ denotes convolution. Now apply an affine transform to point $P$ to obtain $P_{1}\left(X_{1}, Y_{1}, Z_{1}\right)$ where:

$$
\begin{aligned}
& X_{1}=a_{1} X+b_{1} Y+c_{1} Z+d_{1} \\
& Y_{1}=a_{2} X+b_{2} Y+c_{2} Z+d_{2} \\
& Z_{1}=a_{3} X+b_{3} Y+c_{3} Z+d_{3}
\end{aligned}
$$

Alternatively, apply an affine transform to $Q$ first; and then evolve:

$$
\begin{aligned}
& X_{2}=\left(a_{1} x(u, v)+b_{1} y(u, v)+c_{1} z(u, v)+d_{1}\right) \otimes f(u, v) \\
& Y_{2}=\left(a_{2} x(u, v)+b_{2} y(u, v)+c_{2} z(u, v)+d_{2}\right) \otimes f(u, v) \\
& Z_{2}=\left(a_{3} x(u, v)+b_{3} y(u, v)+c_{3} z(u, v)+d_{3}\right) \otimes f(u, v)
\end{aligned}
$$

so: $X_{2}=X_{1}, Y_{2}=Y_{1}$ and $Z_{2}=Z_{1}$. Affine also includes shape preserving transforms.

Theorem II: Let $\mathcal{S}$ be a closed surface and let $\mathcal{H}$ be its convex hull. $\mathcal{S}$ remains inside $\mathcal{H}$ during evolution.

Proof: Since $\mathcal{H}$ is a convex surface, every plane $P$ tangent to $\mathcal{H}$ contains that surface in the left (or right) half-space it creates. Since $\mathcal{S}$ is inside $\mathcal{H}, \mathcal{S}$ is also contained in the same half-space. Now rotate $P$ and $\mathcal{S}$ so that $P$ becomes parallel to the xy plane. $P$ is now described by the equation $z=c$. Since $P$ does not intersect $\mathcal{S}$, it follows that $z_{Q} \geq c$ for every point $Q\left(x_{Q}, y_{Q}, z_{Q}\right)$ on $\mathcal{S}$. Let $S_{\sigma}$ be an evolved version of $\mathcal{S}$. Every point of $S_{\sigma}$ is a weighted average of a subset of points of $\mathcal{S}$. Therefore, $z_{R} \geq c$ for every point $R\left(x_{R}, y_{R}, z_{R}\right)$ on $S_{\sigma}$, and $S_{\sigma}$ is also contained in the same half-space. This result holds for every plane tangent to $\mathcal{H}$; therefore $S_{\sigma}$ is contained inside the intersection of all the left (or right) half-spaces created by the tangent planes of $\mathcal{H}$. It follows that $S_{\sigma}$ is also inside $\mathcal{H}$.

Theorem III: Simple (not self-intersecting) surfaces remain simple during evolution.

Proof: Assume by contradiction that $\mathcal{S}$ is a simple surface that intersects itself during evolution. The location vector of each point of $\mathcal{S}$ is a continuous function of $\sigma$ during evolution; therefore $\mathcal{S}$ must touch itself at point $P$ before self-intersection. Let $S_{\sigma_{0}}$ be such a surface. Consider two neighborhoods $S_{1}$ and $S_{2}$ of $S_{\sigma_{0}}$ that have point $P$ only in 


\section{BMVC99}

common. Hence $S_{1}$ and $S_{2}$ are non-overlapping. Note that $S_{1}$ and $S_{2}$ have the same tangent plane at $P$. Denote this tangent plane by $T$. Recall that the infinitesimal movement during evolution of each point of $S_{1}$ and $S_{2}$ is determined by the equation

$$
\frac{\partial \mathbf{r}}{\partial t}=H n
$$

where $H$ is the mean curvature, $n$ is normal and $t$ is time. Therefore during evolution, every point will move in the direction of the normal vector by an amount equal to the curvature at that point. Similarly, during reverse evolution, every point will move in the opposite direction of the normal vector by an amount equal to the curvature at that point. It follows that if $S_{1}$ and $S_{2}$ are on opposite sides of $T$, after an infinitesimal amount of reverse evolution they will intersect. This is a contradiction of the assumption that $\mathcal{S}$ was simple before touching itself. Assume then that $S_{1}$ and $S_{2}$ are on the same side of $T$. Note that $S_{1}$ and $S_{2}$ can not be overlapping since they would still be overlapping after an infinitesimal amount of reverse evolution, which is also a contradiction of the assumption that $\mathcal{S}$ was simple before touching itself. Let $S_{1}$ be the segment inside $S_{2}$, i.e., the tangent to $S_{2}$ always has $S_{1}$ to the same side. It can be seen that $S_{1}$ has a larger curvature at $P$ than $S_{2}$. Therefore, after an infinitesimal amount of reverse evolution, point $P$ on $S_{1}$ and point $P$ on $S_{2}$ will move in the same direction, but point $P$ on $S_{1}$ will move by a larger amount. It follows that after an infinitesimal amount of reverse evolution, $S_{1}$ and $S_{2}$ will intersect, which is again a contradiction. It follows that $\mathcal{S}$ remains simple during evolution.

\section{Curvature Estimation}

This section presents techniques for accurately estimating Gaussian and mean curvatures at multiple scales on smoothed free-form 3-D surface. Consider a local parametric representation of a 3-D surface $\mathbf{r}=\mathbf{r}(u, v)$ with coordinates $u$ and $v$, where

$$
\mathbf{r}(u, v)=(x(u, v), y(u, v), z(u, v))
$$

Gaussian curvature $K$ exists at regular points of a surface of class $C_{2}$. When $\mathbf{r}(u, v)$ corresponds to semigeodesic coordinates, $K$ is given by:

$$
K=\frac{b_{u u} b_{v v}-b_{u v}^{2}}{x_{v}^{2}+y_{v}^{2}+z_{v}^{2}}
$$

where subscripts denote partial derivatives, and

$$
\begin{aligned}
b_{u u} & =\frac{A x_{u u}+B y_{u u}+C z_{u u}}{\sqrt{A^{2}+B^{2}+C^{2}}} \\
b_{v v} & =\frac{A x_{v v}+B y_{v v}+C z_{v v}}{\sqrt{A^{2}+B^{2}+C^{2}}} \\
b_{u v} & =\frac{A x_{u v}+B y_{u v}+C z_{u v}}{\sqrt{A^{2}+B^{2}+C^{2}}}
\end{aligned}
$$




\section{BMVC99}

where $A=y_{u} z_{v}-z_{u} y_{v}, B=x_{v} z_{u}-z_{v} x_{u}$ and $C=x_{u} y_{v}-y_{u} x_{v}$. Mean curvature $H$ also exists at regular points of a surface of class $C_{2}$. Again, when $\mathbf{r}(u, v)$ corresponds to semigeodesic coordinates, $H$ is given by:

$$
H=\frac{b_{v v}+\left(x_{v}^{2}+y_{v}^{2}+z_{v}^{2}\right) b_{u u}}{2\left(x_{v}^{2}+y_{v}^{2}+z_{v}^{2}\right)}
$$

Both Gaussian and mean curvatures are direction-free quantities, and invariant to rotation and translation of the surface [2]. On smoothed surfaces of 3-D objects, the procedure for estimating the Gaussian and mean curvatures are as follows:

For each point of the surface, $p(x(u, v), y(u, v), z(u, v))$, the corresponding local neighbourhood data is convolved with the partial derivatives of the Gaussian function $G(u, v, \sigma)$,

$$
\begin{gathered}
x_{u}=x * \frac{\partial G}{\partial u}, \quad y_{u}=y * \frac{\partial G}{\partial u}, z_{u}=z * \frac{\partial G}{\partial u} \\
x_{v}=x * \frac{\partial G}{\partial v}, \quad y_{v}=y * \frac{\partial G}{\partial v}, z_{v}=z * \frac{\partial G}{\partial v} \\
x_{u u}=x * \frac{\partial^{2} G}{\partial u^{2}}, \quad y_{u u}=y * \frac{\partial^{2} G}{\partial u^{2}}, \quad z_{u u}=z * \frac{\partial^{2} G}{\partial u^{2}} \\
x_{v v}=x * \frac{\partial^{2} G}{\partial v^{2}}, \quad y_{v v}=y * \frac{\partial^{2} G}{\partial v^{2}}, \quad z_{v v}=z * \frac{\partial^{2} G}{\partial v^{2}} \\
x_{u v}=x * \frac{\partial^{2} G}{\partial u \partial v}, \quad y_{u v}=y * \frac{\partial^{2} G}{\partial u \partial v}, \quad z_{u v}=z * \frac{\partial^{2} G}{\partial u \partial v}
\end{gathered}
$$

where $*$ denotes convolution. Finally, curvature values on a 3-D surface are estimated by substituting these values into equations (1) and (2), respectively.

\subsection{Curvature Zero-crossing Contours}

Having computed curvature values at each vertex of a smoothed 3-D surface, one can locate curvature zero-crossing contours where curvature functions $K$ or $H$ defined by equations (1) and (2), are equal to zero. Curvature zero-crossing contours can be useful for segmenting a smoothed 3-D surface into regions. The process of recovery of the curvature zero-crossing contours is identical for Gaussian and mean curvatures. Every edge $e$ of the smoothed surface is examined in turn. If the vertices of $e$ have the same signs of curvature, then there is no curvature zero-crossing point on $e$. However, if the vertices of $e$ have different signs of curvature, then there exists a point on $e$ at which curvature goes to zero. The zero-crossing point is assumed to be at the midpoint of $e$. The other two edges of the triangle to which $e$ belongs will then be checked since there will be another zero-crossing point on one of those edges. When that zero-crossing is found, it is connected to the previously found zero-crossing. The curvature zero-crossing contour is tracked in this fashion until one arrives back at the starting point.

\subsection{Curvature Error}

Two principle curvatures are defined at each point of a 3-D surface of class $C_{2}$. For two principal curvatures $k_{1}$ and $k_{2}$, the Gaussian and mean curvatures are defined as:

$$
K=k_{1} k_{2}
$$




\section{BMVC99}

and

$$
H=\frac{k_{1}+k_{2}}{2}
$$

Suppose $\tilde{k_{1}}$ and $\tilde{k_{2}}$ are the measured values of $k_{1}$ and $k_{2}$. It follows that $\tilde{k_{1}}=k_{1}+\varepsilon$ and $\tilde{k_{2}}=k_{2}+\varepsilon$, where $\varepsilon$ represents error $(\varepsilon \ll 1)$, then Gaussian curvature is given by,

$$
K=\tilde{k_{1}} \tilde{k_{2}}=k_{1} k_{2}+\varepsilon\left(k_{1}+k_{2}\right)+\varepsilon^{2}
$$

and mean curvature $H$ is given by

$$
H=\frac{\tilde{k_{1}}+\tilde{k_{1}}}{2}=\frac{k_{1}+k_{2}}{2}+\varepsilon
$$

Since $k_{1}$ and $k_{2}$ are very small values (object sizes are quite large to avoid numerical problems) and $\varepsilon\left(k_{1}+k_{2}\right)+\varepsilon^{2} \ll \varepsilon$, it follows that for an error in the values of principal curvatures $k_{1}$ and $k_{2}$, the error introduced in Gaussian curvature is expected to be smaller than that of mean curvature. On smoothed surfaces of 3-D objects, the procedure for curvature estimation error is as follows: For each vertex the curvature values are computed for all directions and the average curvature value is then used as the correct value of curvature for that vertex. Then, the error in direction $i$ is given by,

$$
\text { error }_{i}=\frac{\left|\bar{k}-k_{i}\right|}{|\bar{k}|}
$$

where $k_{i}$ is the curvature value for direction $i$, and $\bar{k}$ is the average curvature value.

\section{Results and Discussion}

\subsection{Curvatures}

This section presents the results of application of our curvature estimation techniques to 3-D objects using methods as described in previous section. The diffusion results for our 3-D surfaces were given in [8]. Complete triangulated models of 3-D objects used for our experiments are constructed at our centre [3]. The first example is a phone handset. After smoothing the object, the Gaussian curvatures of all vertices were estimated. To visualise these curvature values on the surface, they are then mapped to gray levels using the Visualisation Toolkit (VTK) [11], and the results are shown in Figure 1(a). Surface curvature gray levels are coded as follows: white $=$ high, black $=$ low and other gray levels designate in-between values. All convex corners of the phone handset are white, indicating high curvature values, whereas the concave corners are black indicating low curvature values and flat areas are gray since their curvature values are close to zero. The same experiment was repeated to estimate the mean curvatures of the phone handset and the results are shown in Figure 1(b). This indicates that mean curvature values for the edges are different than those for flat areas, as expected. Next, the curvature zero-crossing contours of these surfaces were also found and displayed on the surface using VTK. Figure 1(c) shows Gaussian curvature zero-crossing contours for the smoothed phone handset. Figure 1(d) shows mean curvature zero-crossing contours for the same object.

The next object was a chair. Its Gaussian curvature values were estimated and results are shown in Figure 2(a). These results again confirm that curvature values are high and 


\section{BMVC99}

low at convex and concave corners, respectively, and close to zero on flat regions such as the seat and the back. The mean curvatures of the chair were also estimated and the results are shown in Figure 2(b). Figure 2(c) shows the Gaussian curvature zero-crossing contours for the smoothed chair, and 2(d) shows the mean curvature zero-crossing contours for the same object. Curvature zero-crossing contours can be used for segmenting surfaces into regions. These features can be utilised by later processes for robust surface matching and object recognition with occlusion.

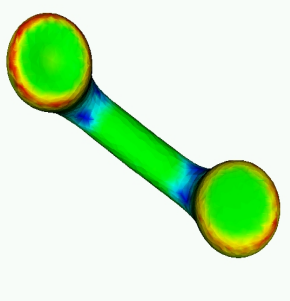

(a) Gaussian

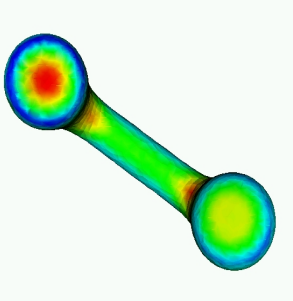

(b) Mean

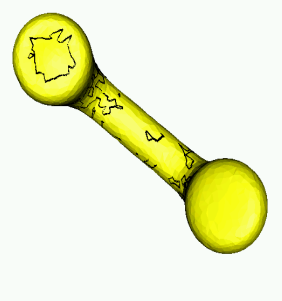

(c) Gaussian

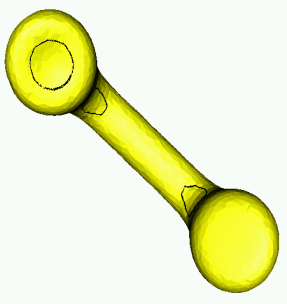

(d) Mean

Figure 1: Curvatures and their zero-crossing contours on the phone handset

\subsection{Curvature Error Estimation}

This section discusses the performance of our technique for curvature estimation when all possible directions at each vertex on a 3-D surface are selected as an arbitrary direction in order to construct the first geodesic line. As mentioned before, in our method the direction of the first geodesic line is randomly selected. We will then examine curvature estimation error for all possible directions. Smoothing results are shown where the direction of one of the incident edges is selected as an arbitrary direction and the surface is also sampled locally at a step size equal to 1000 . The test object was a rabbit with 1996 triangles and 1000 vertices as shown in Figure 3. The ears disappear after 10 iterations and the object becomes a smooth and rounded shape. The smoothing procedure was repeated

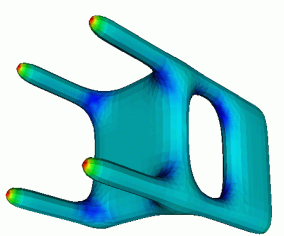

(a) Gaussian

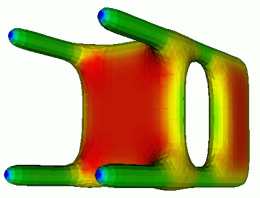

(b) Mean

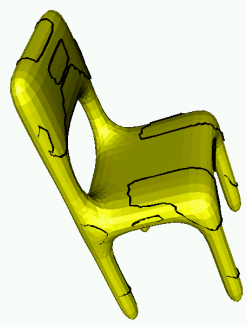

(c) Gaussian

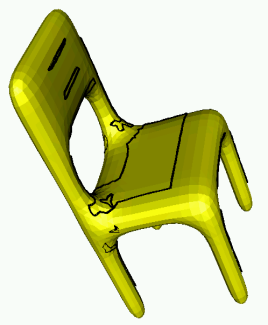

(d) Mean

Figure 2: Curvatures and their zero-crossing contours on the chair 


\section{BMVC99}

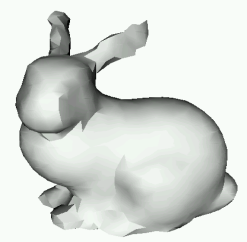

(a) Original

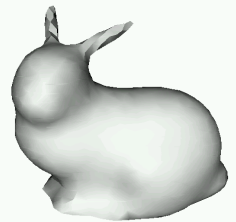

(b) 5 iterations

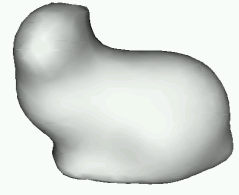

(c) 10 iterations

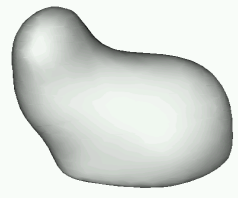

(d) 24 iterations

Figure 3: Diffusion of the RABBIT

where for each vertex all possible directions for the first geodesic line were constructed and different step sizes were considered. After the object was smoothed, the Gaussian and mean curvatures of all vertices were estimated. Then for each vertex the maximum error in curvature values of all possible directions was found. Figure 4(a) shows the maximum error distribution for estimating Gaussian curvatures of the rabbit when all possible directions were selected with the step size varying from 500 to 3000 . These results indicate that the errors were reduced for step sizes between 1000 to 2000 . The error is reduced to about $1.23 \%$, for one iteration. We then repeated the experiment for the minimum error in Gaussian curvature values of all possible directions and results are shown in Figure 4(c). These indicate that for the step sizes between 1000 to 2000 the error is reduced to about $0.96 \%$. When average error in curvature values of all possible directions was estimated, it was found to be about $1.15 \%$ after one iteration, as shown in Figure 4(e). However, as the surface becomes smooth iteratively, the errors reduce as shown in Figure 4. After 24 iterations the maximum, minimum and average errors in curvature values are reduced to $0.92 \%, 0.5 \%$ and $0.7 \%$, respectively.

The above procedures were repeated for estimation of mean curvatures. Figure 4(b), (d) and (f) show the error distribution for estimating mean curvature of the rabbit and these results also indicate that the maximum, minimum and average errors in curvature values are about $2.85 \%, 2.7 \%$ and $2.8 \%$, respectively, for one iteration. After 24 iterations these errors reduce to about $2.1 \%, 1.6 \%$ and $1.9 \%$, respectively. Notice that the errors in the estimation of Gaussian curvatures are lower than that of the mean curvatures as was discussed in the previous section.

Our experiments indicate that estimation of Gaussian and mean curvatures on smoothed surfaces is very accurate and not affected by the arbitrary direction of the first geodesic line when constructing semigeodesic coordinates. Our technique was also applied to incomplete surfaces with the results shown in [17]. Animation of surface diffusion can be observed at: http://www.ee.surrey.ac.uk/Research/VSSP/demos/css3d/index.html.

\section{Conclusions}

A novel technique for multi-scale curvature computation on a smoothed 3-D surface is presented. Complete triangulated models of 3-D objects are constructed and using a local parametrisation technique, are then smoothed using a 2-D Gaussian filter. Some evolution properties of 3-D surfaces were described in this paper. The surface Gaussian and mean curvatures were also estimated. To visualise these on the surface, curvature val- 


\section{BMVC99}
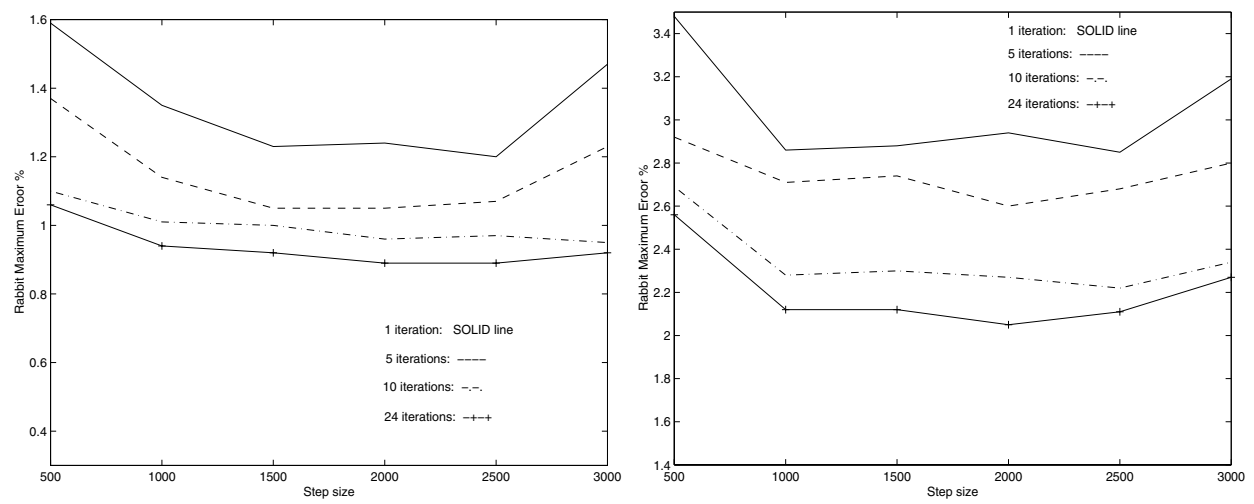

(a) Maximum Error in Gaussian Curvature
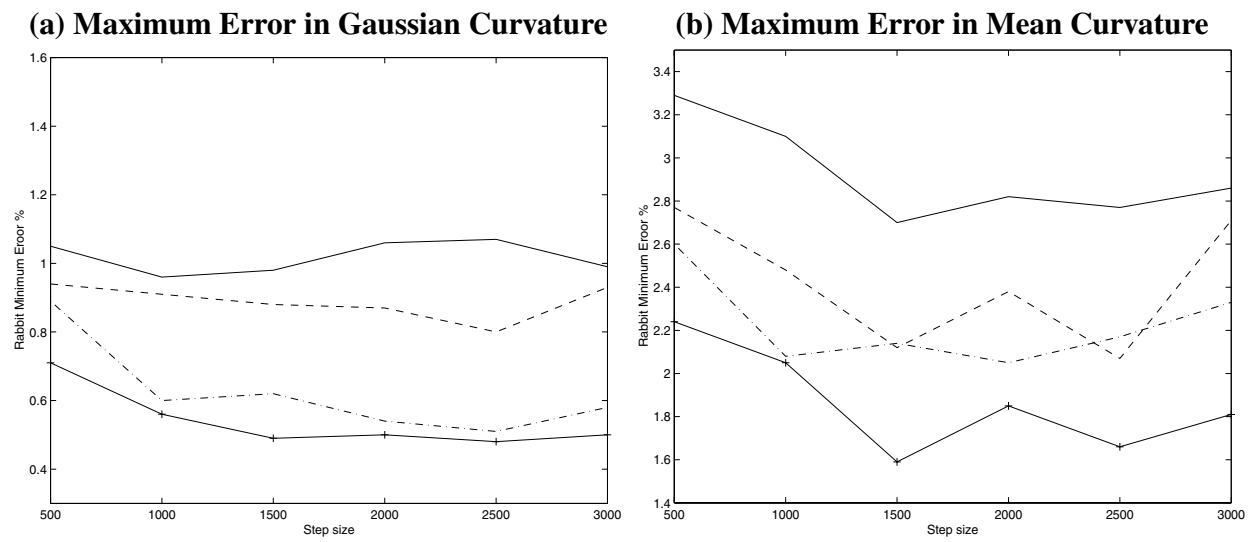

(c) Minimum Error in Gaussian Curvature

(d) Minimum Error in Mean Curvature

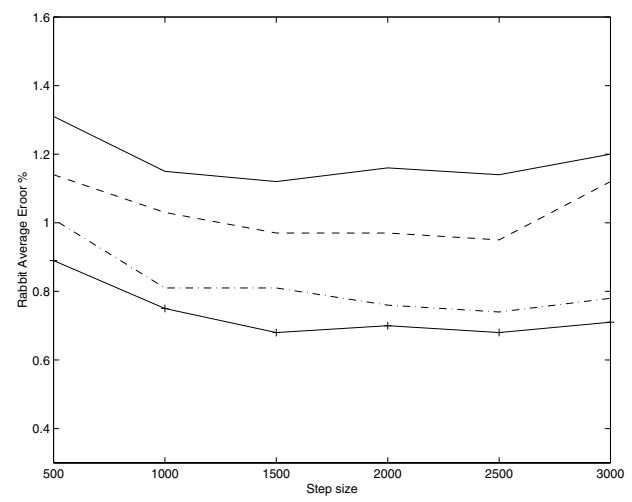

(e) Average Error in Gaussian Curvature

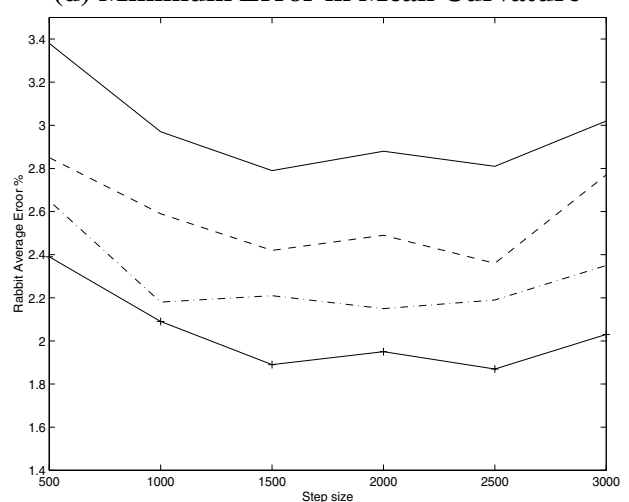

(f) Average Error in Mean Curvature

Figure 4: Gaussian and Mean Curvature Error distributions of the RABBIT 


\section{BMVC99}

ues were mapped to gray levels and shown directly on the surface. Next, the curvature zero-crossing contours were recovered and displayed on the surface.

In our technique semigeodesic coordinates are constructed at each vertex of the mesh which becomes the local origin. A geodesic from the origin is first constructed in an arbitrary direction such as the direction of one of the incident edges. During the diffusion process, 3-D surfaces were also sampled locally using different step sizes. The performance of our technique for curvature estimation when selecting different directions as an arbitrary direction at each vertex was also presented and results indicated that the errors involved in the estimation of Gaussian and mean curvatures were quite small after only one iteration. Furthermore, as the surface became smoothed iteratively, the errors were further reduced for both Gaussian and mean curvatures. These results indicated that estimation of Gaussian and mean curvatures on smoothed surfaces is very accurate and not affected by the arbitrary direction of the first geodesic line when constructing semigeodesic coordinates. It was also shown that the estimation error of Gaussian curvature is less than that of mean curvature.

\section{References}

[1] P J Besl and R C Jain. Three dimentional object recognition. ACM Computing Surveys, 17:75-145, 1985.

[2] A Goetz. Introduction to differential geometry. Addison-Wesley, Reading, MA, 1970.

[3] A Hilton, A J Stoddart, J Illingworth, and T Windeatt. Reliable surface reconstruction from multiple range images. In Proc European Conference on Computer Vision, pages 117-126, Cambridge, UK, 1996.

[4] N Khalili, F Mokhtarian, and P Yuen. Free-form surface description in multiple scales: Extension to incomplete surfaces theoretical results. In 8th International Conference on Computer Analysis of Images and Patterns, Slovenia, Ljubljana, 1999.

[5] J J Koenderink. Solid shape. MIT Press, Cambridge, MA, 1990.

[6] J J Koenderink and A J vanDoorn. Dynamic shape. Biological cybernetics, 53:383-396, 1986.

[7] F Mokhtarian. A theory of multi-scale, torsion-based shape representation for space curves. Computer Vision and Image Understanding, 68(1):1-17, 1997.

[8] F Mokhtarian, N Khalili, and P Yuen. Multi-scale 3-d free-form surface smoothing. In Proc British Machine Vision Conference, pages 730-739, 1998.

[9] F Mokhtarian and A K Mackworth. A theory of multi-scale, curvature-based shape representation for planar curves. IEEE Trans Pattern Analysis and Machine Intelligence, 14(8):789-805, 1992.

[10] M Pilu and R Fisher. Recognition of geons by parametric deformable contour models. In Proc European Conference on Computer Vision, pages 71-82, Cambridge, UK, 1996.

[11] W Schroeder, K Martin, and B Lorensen. The Visualization Toolkit: An Object Oriented Approach to 3-D Graphics. Prentice Hall, 1996.

[12] J A Sethian. Level set methods. Cambridge University Press, 1996.

[13] S S Sinha and R Jain. Range image analysis. In Handbook of Pattern Recognition and Image Processing: Computer Vision (T Y Young, ed.), volume 2, pages 185-237, 1994.

[14] B I Soroka and R K Bajcsy. Generalized cylinders from serial sections. In Proc IJCPR, 1976.

[15] G Taubin. Curve and surface smoothing without shrinkage. In Proc ICCV, pages 852-857, 1995.

[16] G Taubin. Optimal surface smoothing as filter design. In Proc ECCV, 1996.

[17] P Yuen, F Mokhtarian, and N Khalili. Multi-scale 3-d surface desription: open and closed surfaces. In Scandinavian Conference on Image Analysis, pages 303-310, Greenland, 1999. 